\title{
A Contact Force Sensor based on S-shaped Beams and Optoelectronic Sensors for Flexible Manipulators for Minimally Invasive Surgery (MIS)
}

\author{
Yohan Noh, Sangjin Han, Pawel Gawenda, Wanlin Li, Sina Sareh, Kawal Rhode
}

\begin{abstract}
Flexible, highly articulated robotic tools can greatly facilitate procedures in which the operator needs to access small openings and confined spaces. Particularly, in the context of robotic-assisted minimally invasive surgery (RMIS), the application of such manipulation tools can be significantly beneficial in preventing unnecessary interactions with sensitive body organs by which reducing patient's recovery time when compared with conventional methods. However, these systems usually lack tactile feedback and are not able to perceive and quantify the interactions between themselves and soft body organs. This deficiency may result in damaging the organs due to unwanted excessive force applied. To this end, we introduce a contact force sensor based on three 'dyadic-S-shaped' beams and three optoelectronic sensors. The modular design of a flexible manipulation system described as part of this paper allows ready integration of a series of the proposed sensors within its structure. The sensor uses our novel sensing principle for measuring contact forces. The strategic employment of custom sensor structure and the optoelectronic components fulfill our design objectives which has been focused on the creation of a modular, low-cost, low-noise (electrically) with large voltage variation, without the need for an amplifier, through a simple fabrication process for MIS. Our experimental results, following a very simple calibration processes show the average errors of $F_{x}(+19.37 \% \pm 0.82,-18.32 \% \pm 2.06)$ and $F_{y}(+18.56 \% \pm 1.69$, $17.00 \% \pm 1.32)$, and the average RMS errors of $F_{x}(0.12 \mathrm{~N} \pm 0.0067)$ and $F_{y}(0.11 \mathrm{~N} \pm 0.0032)$ in the measurement of force values within the range of -4 to $4 \mathrm{~N}$.

Keywords-MIS; optoelectronic sensor; contact force; force sensor
\end{abstract}

\section{INTRODUCTION}

Minimally Invasive Surgery (MIS) provides low invasiveness to the patient's body and enhances the robustness of regular medical surgeries [1-2]. MIS procedures involve operations in which there are incisions up to $15 \mathrm{~mm}$ in diameter at the skin level and surgical tools are inserted in the form of laparoscopes or articulated devices such as robotic manipulation arms. This approach guarantees that patients are

Manuscript received $17^{\text {th }}$ March, 2019, revised $26^{\text {th }}$ Aug, 2019, and revised $29^{\text {th }}$ Sep, 2019.

The research leading to these results has received funding from BEng project 2017-2018, Department of Biomedical Engineering, King's College London.

Yohan Noh is with the Department of Biomedical Engineering, King's College London, UK, and the Department of Mechanical and Aerospace Engineering, Brunel University London, UK (e-mail corresponding author: yohan.noh@brunel.ac.uk).

Pawel Gawenda and Kawal Rhode are with the Department of Biomedical

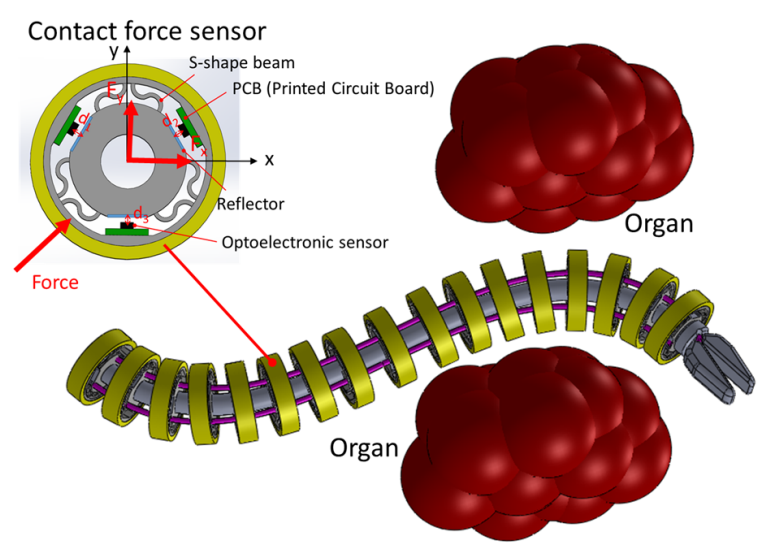

Figure 1. Contact force sensor incorporated into a flexible robotic manipulator for MIS.

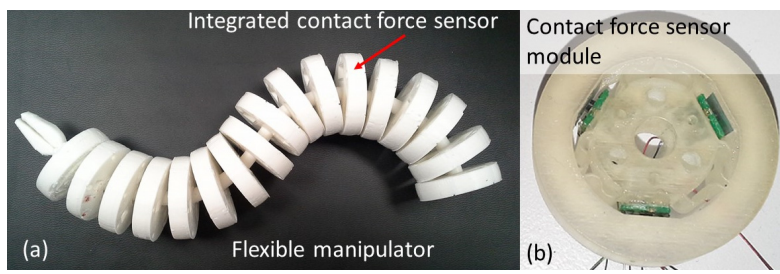

Figure 2. The configuration of the flexible manipulator (a), integrating an array of contact force sensors (b).

at lower risk to fatal conditions and recover in a shorter period of time.

The Da Vinci Surgical System [3-4] is one of the most popular technologies used in RMIS. Its precise movement and enhanced vision using high-resolution dual cameras assist surgeons to carry out surgical operations in a highly safe and comfortable manner [5-6]. In what follows, the advent of flexible manipulators has been proposed to overcome the limited workspace of rigid surgical instruments. The flexible manipulators allow us to access narrow and small openings, intricate pathways and blind spots [7-8]. Flexible manipulators are classified into two main groups according to their actuation principle: extrinsic or intrinsic.

Engineering, King's College London, UK, and the Department of Mechanical and Aerospace Engineering, Brunel University London, UK.

Sangjin Han is with U. S. Army Research Laboratory, Adelphi, Maryland and Booz Allen Hamilton Inc. McLean, Virginia, U.S.A.

Sina Sareh is with the School of Design, Royal College of Art, London, UK.

Wanlin Li is with Department of Electronic engineering and Computer Science, Queen Mary University of London, UK. 
As an extrinsic actuation approach, Kahrs et al. developed a stiffness controllable flexible manipulator using a tendon driven mechanism that is not only able to change its shape but also it can elongate [9-10]. Other prominent examples of extrinsic actuation approaches include the CardioARM; a highly articulated manipulation system that can provide unlimited and controllable flexibility using a tendon drive mechanism [11]; Hansen Medical Inc. proposed a catheter robot by integrating a multi-axis force/torque sensor at the tip of the multi-backbone structure as well as shape control capability [12]; Webster III et al. created a concentric tube robot to reach a specific location of the brain with no damage [13].

A number of researchers have proposed intrinsic actuation approaches, important examples include the i-Snake based on micro-motors [14], a hydraulically or pneumatically actuated stiffness controllable soft continuum flexible manipulator integrating heterogenous sensing modalities: tactile, force/torque and shape detection sensors for MIS [15-17]; an alloy-actuated shape memory concentric manipulator for percutaneous needle-based procedures such as prostate biopsy and breast biopsy to guide it to the target [18]. These are considered as the intrinsic actuation approaches.

Despite all of the remarkable progress in the development for the flexible manipulators, sensing modalities which recognise physical conditions in the body and/or which detects the manipulator's shape and posture have not been fully realised. Whereas legacy manipulators provide their location information to some extent, they do not feedback information about touch and precise force exertion, which are critical for protecting vital organs against potential damages [19-20].

High-resolution 3D dual endoscopic cameras [21-22] in the medical instruments enable surgeons to visually inspect physical conditions of the surgical environment in the body. However, it is not always the case when the cameras are hindered by other adjacent instruments or organs. Moreover, dynamic shape changes of the manipulators cannot be visually captured and confirmed in real time since the cameras deliver images in the direction to which the tip of the instrument is headed. Thus, the manipulator may damage soft tissues that are not caught by the cameras.

Recently, state-of-the-art flexible manipulators [23-28] have integrated various sensing modalities such as shape and stretch sensing units, tactile sensing units and multi-axis force/torque sensors. For example, the continuum manipulators are integrated with light intensity modulation based fibre optic sensors to measure force/torque [19, 23] as well as the manipulators' shape [29] based on stretch sensing elements [49]. The fibre-optic sensors can be designed to provide a high sensing resolution and sensitivity, at a relatively low-cost. Since fibre-optic sensors use no electrical component in the sensing site, they are compatible with Magnetic Resonance Imaging (MRI) environments. However, it is typically difficult to miniaturise the overall size of the manipulator due to the space needed to house the optical fibres. The Fibre Bragg Grating (FBG) sensing elements are widely used to measure contact forces and the shape of the flexible manipulators [25-27]. This element helps to cut down the size of the manipulator without the loss of outstanding sensing
TABLE I. MEASURABLE ForCE RANGE AND SENSOR STRUCTURE CONFIGURATION

\begin{tabular}{|c|c|c|c|}
\hline \multicolumn{2}{|c|}{ Force Range } & $\begin{array}{c}\text { Sensor structure } \\
\text { configuration }\end{array}$ & $\begin{array}{c}\text { Sensor structure } \\
\text { Material property }\end{array}$ \\
\hline$F_{x}$ & $+/-4 \mathrm{~N}$ & $\begin{array}{c}H=7.5 \mathrm{~mm} \\
D=26.5 \mathrm{~mm}\end{array}$ & $\begin{array}{c}\text { Tensile modulus of } 1283 \mathrm{MPa}, \\
\text { Mass density of } 1020 \mathrm{~kg} / \mathrm{m} 3, \\
\text { Yield strength of } 42500000 \mathrm{~N} / \mathrm{m} 2\end{array}$ \\
\cline { 1 - 2 }$F_{y}$ & $+/-4 \mathrm{~N}$ & $\begin{array}{c}r=1.0 \mathrm{~mm} l=0.45 \mathrm{~mm} \\
t_{l}=0.4 \mathrm{~mm}, t_{2}=7.5 \mathrm{~mm}\end{array}$ & \\
\hline
\end{tabular}

modalities. Nevertheless, it is fragile to temperature variation and it is of high cost due to the interrogator which reads off data from the FBG sensing elements [30].

In this paper, we introduce a novel contact force sensor based on light intensity sensing principle using optoelectronic sensors. The size and shape are designed such that this can be integrated into the flexible manipulators in practice. Since 2012, the authors have initiated the development of soft/flexible continuum robotic arms at King's College London. The arms have been initially integrated with shape and three axis force/torque sensing units that use optical fibres and optoelectronics and work based on the light intensity modulation principle [15-17, 19-20, 23-24]. While, the shape sensing units, consisting of optical fibres and fibre-optic sensors, measure the manipulator's shapes, the three-axis force/torque sensor, embedding the optoelectronic sensing elements, can effectively detect the external forces/torques $\left(F_{z}\right.$, $\left.M_{x}, M_{y}\right)$ applied to specified locations on the arm. Although both earlier methods of sensing show outstanding capability to estimate the manipulator's shapes and the external forces/torques, they were limited in estimating arm's complex, non constant-curvature, shapes; the force/torque sensors were originally designed for integration only in the joints between the segments of the soft arm, assuming that fusing the joint force/torque and shape sensor information, which was using a constant-curvature approximation, would be sufficient for control purposes. However, our later experimental tests indicated the need for improvement of the sensing system, as in practice the arm can undergo non constant-curvature shapes. Therefore, our recent efforts has been focused on the development of a more distributed and modular sensing system, as described in this study and shown in Figs. 1-2, integrating an array of contact force sensors and shape sensing units, since 2017, [31-33].

The flexible manipulators consist of a series of identical segments through which deformable (elastic) cylindrical beams and wires are passed. The goal of our project is to integrate two sensing units measuring contact forces -- $F_{x}$ and $F_{y}$, and two relative orientations -- roll and pitch, into every successive segment of the flexible manipulator [31-32]. This structure can measure contact force components in every segment and estimate arbitrary shapes using orientation information. Conventional shape sensing methods employing fibre optic and FBG assume constant curvature model [25-27] whereas our approach does not need such an assumption since an arbitrary shape can be estimated by composition of a series of relative orientation matrices. Consequently, this may achieve precise closed-loop motion control as well as acquisition of accurate contact force information [31-33]. 
This paper is organised as follows. We explain the design method and fabrication details of the sensing unit and its structure in Section II. In Section III we present how to calibrate the sensing modalities. In Section IV, we discuss the results of the experiments in terms of accuracy, size, force range and performance.

\section{DESIGN METHODS AND FABRICATION}

\section{A. Design Specifications}

The design specifications for our proposed flexible manipulator are listed in the following:

1) Sensing mechanism adopts optoelectronics technology which requires simple peripheral circuits. Thereby the overall size of the contact force sensor is miniaturised to be fitted into flexible manipulators.

2) This contact force sensor will be simply integrated into all the segments of the flexible arm and, hence, the arm is provided with an ample space to pass electric wires through the flexible manipulator.

3) Each segment has a separate sensing arrangement and is able to measure two components of external force, namely $F_{x}$ and $F_{y}$, the range of which in each direction is from 0 $\mathrm{N}$ to $20 \mathrm{~N}$ [34- 36].

4) The sensor's mechanical structure can be easily fabricated using 3D printing technologies.

5) The outer diameter is about $15 \mathrm{~mm}$ which is the maximum diameter of commercially available trocars in the market.

To verify the principle of the contact force sensor, force
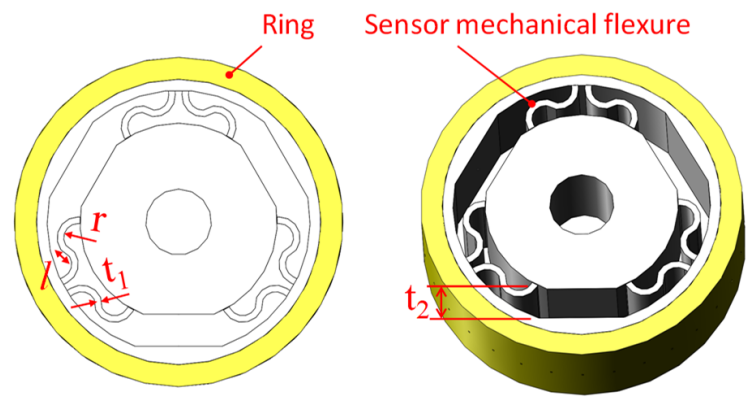

Figure 3. Original design of contact force sensor using two different materials and design parameters for force measurement ranges

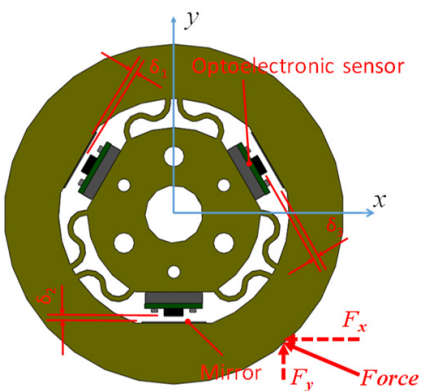

(a)

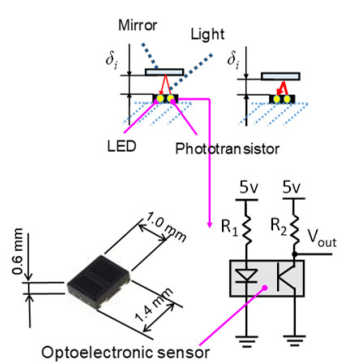

(b)
Figure 4. Configuration of contact force sensor using three double Sshaped beams, three optoelectronic sensors, and three mirrors measurement range, size, and sensor structure material property of our first prototype contact force sensor are summarized in Table I, although its size and measurable range are not satisfied with the design specification. In the following sections, we explain our design methodology to show feasibility of the concept.

\section{B. Configuration of Contact Force Sensor}

The original design of the contact force sensor comprised of two different stiff structures: a ring and the sensor mechanical structure as depicted in Fig. 3. The ring's stiffness is much larger than the one of the sensor's mechanical structure to obtain large deformations of the sensor mechanical structure when external forces are applied. In this study, as a similar approach, a modified sensor structure (diameter $\mathrm{D}=26.5 \mathrm{~mm}$ and height $\mathrm{H}=7.5 \mathrm{~mm}$ ) is proposed to facilitate large deformations of the sensor mechanical flexure as seen in Fig. 4. It is fabricated using a HD Project 3000 rapid prototyping machine, 3D Systems Inc., USA. It should be mentioned that printing one sensor unit costs around $£ 1$ using this approach.

The sensing system is composed of three optoelectronic sensors (NJL5901R-2, $1.0 \times 1.4 \times 0.6 \mathrm{~mm}^{3}$, by New Japan Radio Co., Ltd., Tokyo, Japan), three mirrors as reflectors and three dyadic S-shape beams as elastic mechanical flexures. The mirrors are made using silver-covered reflective surface tapes. The tapes have high reflectance and are adhesive. The tape sticks to the sensor mechanical flexure.

In order to determine force components $F_{x}$ and $F_{y}$, three distances, denoted as $\delta_{1}, \delta_{2}$, and $\delta_{3}$ are measured as illustrated in Fig. 4 (a). Fig. 4 (b) illustrates how deformation information is acquired using an LED and a phototransistor in the optoelectronic sensor (top) and the dimension of the optoelectronic sensor with circuit diagram (bottom) is shown. The LED emits light with consistent intensity and the phototransistor interpret intensity of the received light from mirror into voltage variation [37-39]. When deformation takes place by an external force and a round trip distance between the mirror and the sensor becomes shorter, output voltage in the sensor increases.

The sensor structure has mainly two rings: inner and outer rings. Three dyadic S-shape beams support the gap between two rings. Three mirror-sensor pairs are placed in the outerinner rings, respectively. The beams and mirror-sensor pairs are radially placed one after the other with the same distance and angle. When external force is applied to a point on the outer ring, the distances $\delta_{1}, \delta_{2}$, and, $\delta_{3}$ are measured using optoelectronic sensors in terms of the voltage variations and we estimate direction and magnitude of the force using three distance information.

\section{Sensor Structure Design}

Three dyadic S-shape beams are designed and employed in the structure to estimate the contact force applied to the flexible manipulator. The three dyadic S-shape beams constrain the deformation of the sensor mechanical flexure along the z-axis, caused by the torque component $T_{z}$, thereby allowing only its deformation translationally in the $\mathrm{x}$ and $\mathrm{y}$ directions as shown in Fig. 4. In addition, regardless of 


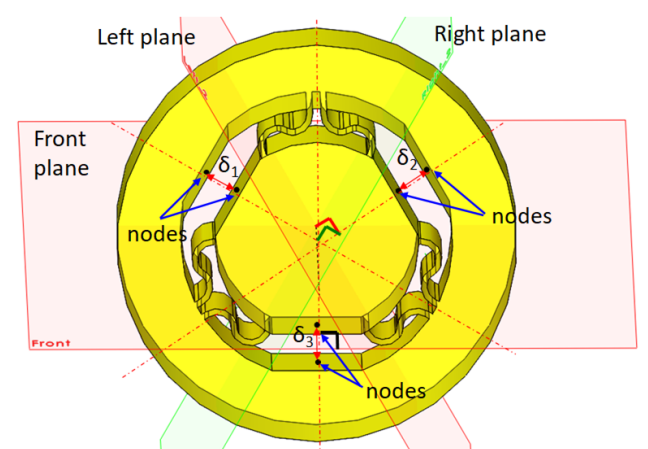

Figure 5. Defined six nodes and three planes to measure three distances $\left(\delta_{1}, \delta_{2}\right.$, and $\left.\delta_{3}\right)$ for FEM SOLIDWORKS Simulation

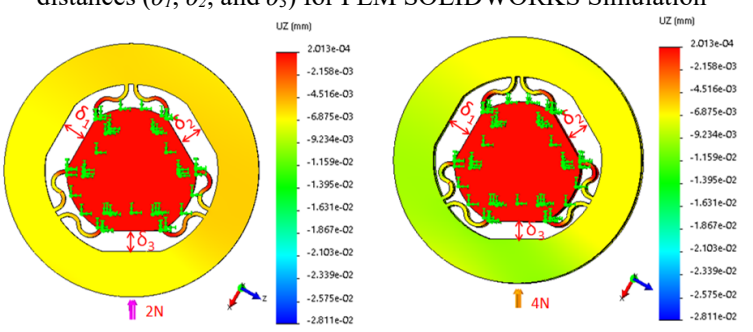

(a)

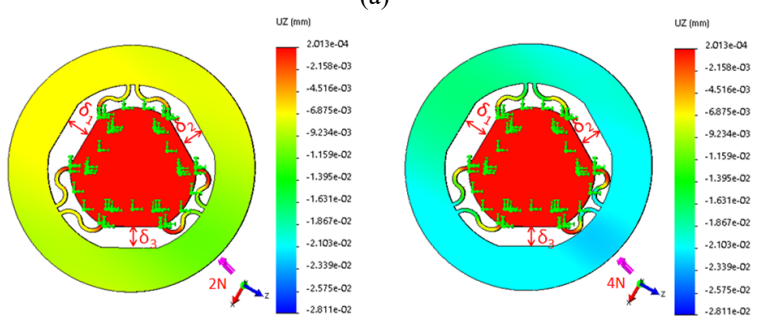

(b)

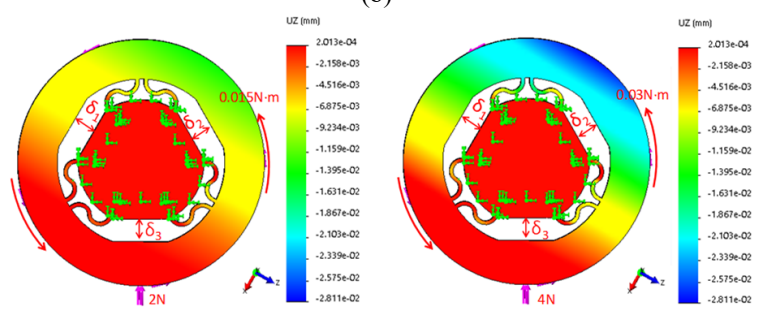

(c)

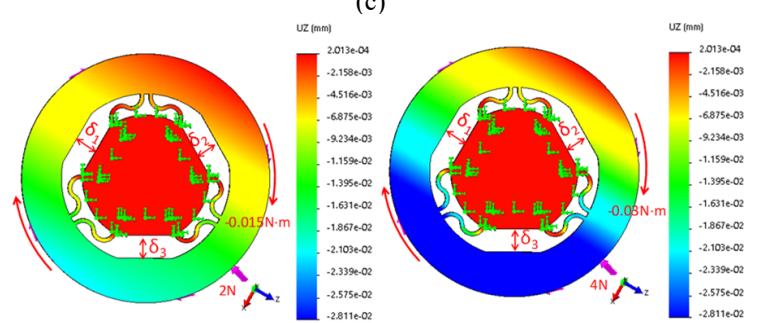

(d)

Figure 6. FEM Simulation performed with the Solidworks Simulation tool: simulation conditions are as follows:

a) $F_{x}, F_{y}, T_{z}:(0 \mathrm{~N}, 2 \mathrm{~N}, 0 \mathrm{~N} \cdot \mathrm{m})(\mathrm{left}),(0 \mathrm{~N}, 4 \mathrm{~N}, 0 \mathrm{~N} \cdot \mathrm{m})($ right $)$

b) $F_{x}, F_{y}, T_{z}:(-1.414 \mathrm{~N}, 1.414 \mathrm{~N}, 0 \mathrm{~N} \cdot \mathrm{m})($ left $),(-2.828 \mathrm{~N}, 2.828 \mathrm{~N}, 0 \mathrm{~N} \cdot \mathrm{m})$ (right)

c) $F_{x}, F_{y}, T_{z}:(0 \mathrm{~N}, 2 \mathrm{~N}, 0.015 \mathrm{~N} \cdot \mathrm{m})(\mathrm{left}),(0 \mathrm{~N}, 4 \mathrm{~N}, 0.03 \mathrm{~N} \cdot \mathrm{m})($ right $)$

d) $F_{x}, F_{y}, T_{z}:(-1.414 \mathrm{~N}, 1.414,-0.015 \mathrm{~N} \cdot \mathrm{m})(\mathrm{left}),(-2.828 \mathrm{~N}, 2.828 \mathrm{~N},-0.03 \mathrm{~N} \cdot \mathrm{m})$ (right)

internal force produced by friction and tendons, the sensor structure can only measure the amount of the applied contact force. The proposed sensor structure should be verified by FEA (finite element analysis) whether it can solely measure contact force components ranging from 0 to $4 \mathrm{~N}$ regardless of applying torque component $T_{z}$.

We point out that the measurement range in Table I can be altered/devised by tuning parameters such as radius $r$ and length $l$, thickness $t_{1}$, width $t_{2}$ indicated in Fig. 3, as well as by using materials with different Young's modulus for making the internal sensor structure.

\section{Sensor Structure Simulation}

To simulate the contact force sensor under different force/torque conditions using SOLIDWORKS FEM simulation, three planes (front, left, right) and six nodes (three on the outer ring and three on the inner ring) are defined as shown in Fig. 5. After each FEM simulation with different force/torque conditions on the contact force, each of the three distances $\left(\delta_{1}, \delta_{2}\right.$, and $\delta_{3}$ in Fig. 5) were measured. Figure 6 shows the results of 8 different force/torque conditions out of 34 combinations for the FEM simulations. In the SOLIDWORKS FEM simulation, the following material properties are used: tensile modulus of $1283 \mathrm{MPa}$, mass density of $1020 \mathrm{~kg} / \mathrm{m}^{3}$, yield strength of $42500000 \mathrm{~N} / \mathrm{m}^{2}$; this information were provided by PROJET VisiJet ${ }^{\circledR}$ EX200 datasheet, 3D SYSTEM Co., Ltd.

Then, a decoupling calibration matrix $\boldsymbol{k}_{\boldsymbol{\delta}}$ representing the relationship between deflections $\left(\delta_{1}, \delta_{2}\right.$, and $\left.\delta_{3}\right)$ and force components $\left(F_{x}\right.$ and $\left.F_{y}\right)$ was calculated using Multi Linear Regression (Eq. 1) [40]. Note that the deflection and force data sets were obtained from the FEM simulations. Subsequently, arbitrary amounts of force $\left(F_{x}\right.$ and $\left.F_{y}\right)$ and torque $T_{z}$ are applied on the contact force sensor in the simulation environment as the same simulation as done in Fig. 6 , and the measured deflections are multiplied by the decoupling calibration matrix to verify the accuracy of the calculated decoupling matrix in estimating the force components (Fig. 7).

The verification simulation results show that the proposed sensor system estimates precise contact force components $F_{x}$ and $F_{y}$ from the three distances information with a maximum of $3.0 \%$ error including crosstalk. In Fig. 7 , we compare $F_{x}$ and $F_{y}$ data from the simulation.

$$
\left[\begin{array}{l}
F_{x} \\
F_{y}
\end{array}\right]=\boldsymbol{k}_{\boldsymbol{\delta}} \cdot \delta=\left[\begin{array}{lll}
k_{\delta 1,1} & k_{\delta 1,2} & k_{\delta 1,3} \\
k_{\delta 2,1} & k_{\delta 2,2} & k_{\delta 2,3}
\end{array}\right] \cdot\left[\begin{array}{l}
\delta_{1} \\
\delta_{2} \\
\delta_{3}
\end{array}\right]
$$

\section{E. Optimization of Two Resistors of Optoelectronic Sensors}

In order to use optoelectronic sensors as parts of the force sensing system, the sensors should produce sufficiently large voltage variations in response to small deformations, since the sensor mechanical flexure nearly behaves as a rigid body. Moreover, the voltage should vary linearly with respect to distance between the optoelectronic sensor and the corresponding mirror in the operating range of forces. Since the amount of deformation in terms of distance is linearly dependent to the external force applied in the operating range, we then have a linear relationship between voltage and force variation.

The optoelectronic sensors have two resistors, namely $R_{1}$ and $R_{2}$ as labelled in the circuit diagram of the sensor in Fig. 

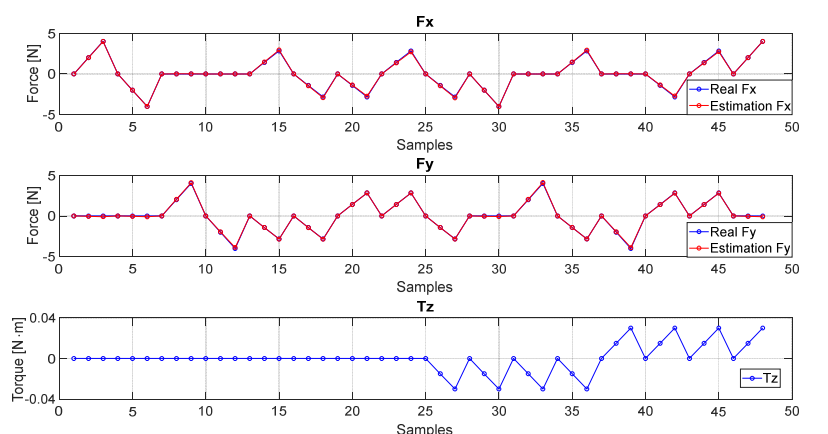

Figure 7. Mechanical structure verification tests of the contact force sensor conducted by FEM Simulation on the Solidworks.

Red: estimated $F_{x}$ and $F_{y}$ from $k_{\delta}$ in Eq. (1), Blue: real $F_{x}, F_{y}$, and $T_{z}$ applied on the Solidworks

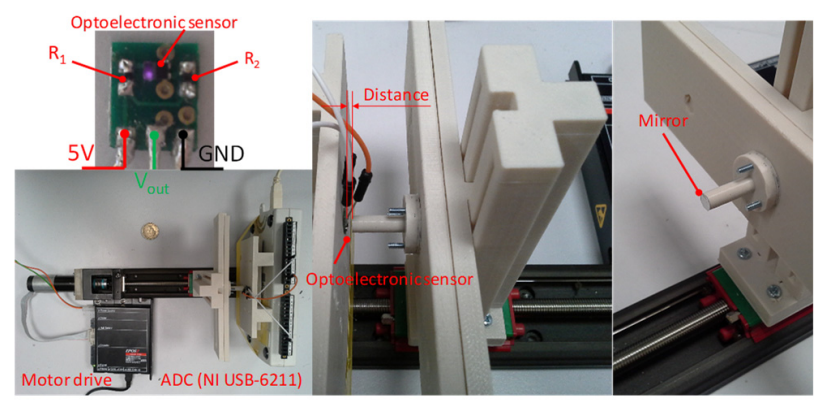

Figure 8. An experiment setup for optimising two resistors $\mathrm{R}_{1}$ and $\mathrm{R}_{2}$ for an optoelectronic sensor

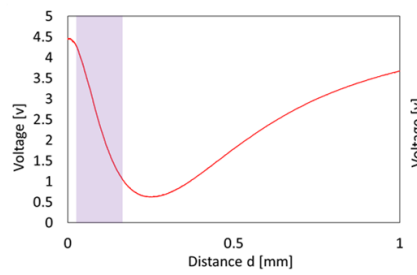

(a)

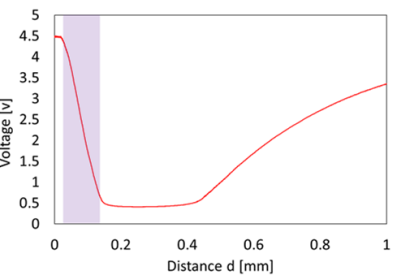

(b)
Figure 9. Characteristic curves of the output voltage of an optoelectronic sensor in case of two conditions:

(a): $R_{l}=1.2 \mathrm{k} \Omega, R_{2}=10 \mathrm{k} \Omega$

(b): $R_{1}=1.0 \mathrm{k} \Omega, R_{2}=10 \mathrm{k} \Omega$

4(b) (bottom). In general, different values of $R_{1}$ and $R_{2}$ induce different characteristic curves in terms of distance and output voltage $V_{\text {out }}$. Thus, we optimise the values of $R_{1}$ and $R_{2}$ through a set of experiments.

\section{1) Optimisation of Two Resistors $R_{1}$ and $R_{2}$}

\section{1) Experiments Setup}

In order to read the sensing voltage, an optoelectronic sensor is interfaced with an analog-digital converter (ADC) board USB-6211, National Instrument, Texas, USA. At the end of a motorised linear guide, a mirror is attached facing the optoelectronic sensor as shown in Fig. 8. The motorised linear guide adjusts the distance between the mirror and the optoelectronic sensor while the distance information is recorded by the encoder of the motor actuator.

\section{2) Experimental Results}

The sensor's characteristic curve when $R_{1}=1.2 \mathrm{k} \Omega$ and $R_{2}$ $=10 \mathrm{k} \Omega$ is shown in Fig. 9 (a). When the mirror is very close to the sensor the voltage drops from $4.5 \mathrm{~V}$ to $0.5 \mathrm{~V}$ rapidly with a small change of distance. However, the curve is not as linear as desired in our distance range of interest, which is shaded in purple.

When $R_{1}=1.0 \mathrm{k} \Omega$ and $R_{2}=10 \mathrm{k} \Omega$, the characteristic curve shows more linear variation of voltage within shorter distance range as shown in Fig. 9 (b). In fact, the voltage variation was nearly $4 \mathrm{~V}$ within about $0.1 \mathrm{~mm}$ distance range. This meets our requirements for the force sensor and no amplifier is needed.

\section{SENSOR CALIBRATION}

In order to find the relationship between the sensor output voltage and the force components $F_{x}$ and $F_{y}$, one can apply different forces and torques through applying physical weights [41-42]. This conventional method requires high precision of the calibrating device and long duration of the calibration process to identify accurate and persistent relationships. We propose a simple and fast calibration method using a force/torque sensor, ATI Nano17, ATI Industrial Automation, Inc., USA [43].

\section{A. Calibration Process with a Calibration Device}

The proposed contact force sensor is installed on a calibration device, as shown in Fig. 10. The calibration device is supported by a mounting base, the ATI Nano 17 sensor sits on the base and the load fixture is then put on the ATI Nano 17 sensor. Our proposed contact force sensor is bolted on the load fixture. The ATI Nano17 sensor features maximum errors of $F_{x}, F_{y}, F_{z}, T_{x}, T_{y}, T_{z}: 1.00 \%, 1.00 \%, 1.00 \%$, $1.50 \%, 1.50 \%, 1.75 \%$, respectively. The calibration device uses a USB-6211 data acquisition board to record the output voltages of three optoelectronic sensors. Data from the ATI Nano 17 are used as a ground truth.

The external forces can be classified into two types: (1) perpendicular to the outer ring (directing the centre of the sensor structure), (2) off-centre by a distance, $d$, but parallel to the direction of (1). See the force and torque arrows in Fig. 10. Using a long stick with a rubber tip, an external force for both types is exerted along the outer ring of the contact force sensor at different angles every 30 seconds ( 2 seconds of applying force plus 28 seconds of rest to ensure the collected data is independent from existing hysteresis effects in plastic sensor structure).

\section{B. Multiple Linear Regression and Sensor Accuracy}

Multiple linear regression finds a linear map between multiple input variables and multiple output variables by fitting a set of linear equations into the observations [40]. In our particular case, each voltage output from an optoelectronic sensor is mapped to a linear combination of the force components $F_{x}$ and $F_{y}$. The relationship can be written by 


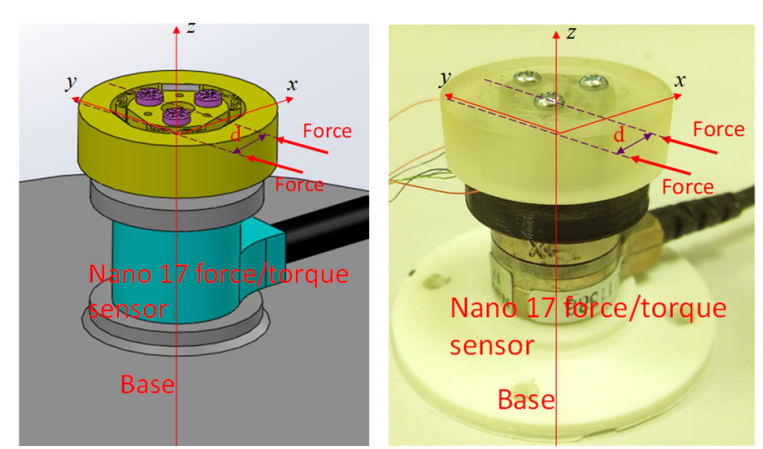

Figure 10. An experimental setup the calibration of $F_{x}$ and $F_{y}$ : external forces are applied to the contact force sensor

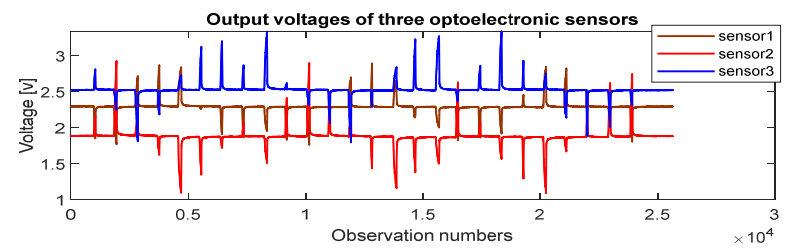

Figure 11. The output voltages of the three optoelectronic sensors while a variety of force/torque conditions are exerted on the contact force sensor.
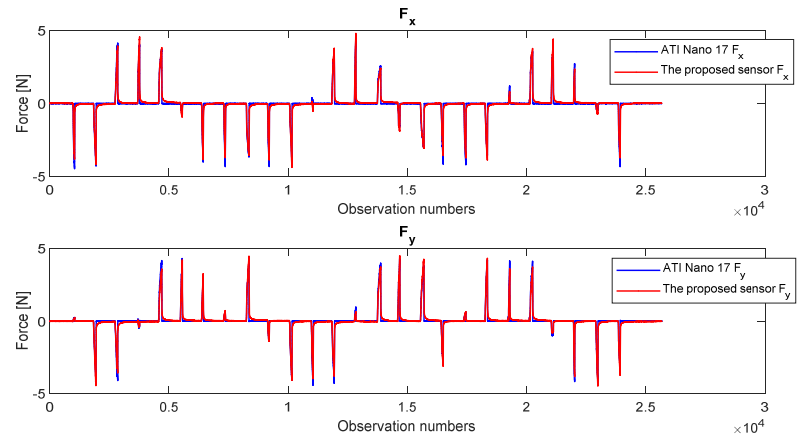

Figure 12. Comparison between ATI Nano 17 and our contact force sensor.



Figure 13. Torque $T_{z}$ data while a variety of force/torque conditions are exerted on the contact force sensor $\left(F_{x}\right.$ and $F_{y}$ force data as shown in Fig. 12 (red line).

$$
\left[\begin{array}{l}
F_{x} \\
F_{y}
\end{array}\right]=\boldsymbol{k}_{\boldsymbol{v}} \cdot \delta=\left[\begin{array}{lll}
k_{v 1,1} & k_{v 1,2} & k_{v 1,3} \\
k_{v 2,1} & k_{v 2,2} & k_{v 2,3}
\end{array}\right] \cdot\left[\begin{array}{l}
v_{1} \\
v_{2} \\
v_{3}
\end{array}\right]
$$

where $\boldsymbol{k}_{\boldsymbol{v}}$ is a linear map, $v_{1}, v_{2}$ and $v_{3}$ are output voltages from optoelectronic sensors and $F_{x}, F_{y}$ are the force components. Using the data from the previous calibration, we determine $\boldsymbol{k}_{\boldsymbol{v}}$, called decoupling calibration matrix, as follows:

$$
\boldsymbol{k}_{\boldsymbol{v}}=\left[\begin{array}{llc}
4.4871 & -2.7371 & -3.7895 \\
1.3913 & -2.2686 & 5.0611
\end{array}\right]
$$

Now, we verify the accuracy of the decoupling calibration matrix. A total of 5 experiments are carried out. In each experiment, external forces are exerted on the contact force sensor in the similar manner to the calibration process. The estimated force components, $F_{x}$ and $F_{y}$, are calculated by multiplying $\boldsymbol{k}_{\boldsymbol{v}}$ in Eq. (3) with the output voltages of the optoelectronic sensors. For instance, the output voltages are shown in Fig. 11. The results of $F_{x}$ and $F_{y}$, estimated values using $\boldsymbol{k}_{\boldsymbol{v}}$ and the ground truth from ATI Nano 17 are compared in Fig. 12. The result shows that the calibration matrix could estimate the force components accurately, despite nonzero torque values, $T_{z}$, as shown in Fig. 13 (note that Figs.11-13 shows one of the outcomes out of the 5 experiments).

The error statistics (averaged error values and standard deviation over 5 experiments) are summarised in Table II. It can be seen that the decoupling calibration matrix follows the ground truth in force measurements over $-4 \sim 4 \mathrm{~N}$ range with mild errors.

\section{DISCUSSIONS}

\section{A. Accuracy of Contact Force Sensor}

The conceptual idea of this study is that the dyadic-Sshaped beam retains translational deformation while restraining rotational deformation. The simulation results in Fig. 7 suggests that the beam structure estimates force/torque values precisely in spite of torque components. In our experiments, however, the measured distances are generally perturbed by a surface angle even if the absolute distance is same. The calibration matrix might have less accuracy due to some inconsistent data. As a future work, a new sensor mechanical structure will be improved so that the rotational motion is fully eliminated in the deformation, leaving pure translational motion.

Our proposed sensor has large hysteresis since the sensor structure is made of plastic with the same properties used in the simulation studies in Section II-C. An example of large hysteresis in the output voltages of three optoelectronic sensors are shown in Fig. 11. In comparison with force components measured from ATI Nano 17, the force components from the proposed sensor lags and these lagging phenomena are due to the hysteresis as shown in Fig. 12. Since this hysteresis effect is included when the decoupling calibration matrix is calculated, the accuracy of the decoupling calibration matrix can be deteriorated by large hysteresis. To avoid unwanted hysteresis for more accurate calibration data, a metal structure fabricated by a 3D metal printer can be applied. Current cutting-edge technologies of $3 \mathrm{D}$ metal printing can guarantee a high-resolution printing quality $(0.1 \mathrm{~mm})$ at low cost (one unit is around $£ 20$ ) [44].

If the structure uses metal, then the deformation range of interest will be greatly reduced. Hence, the resistors $R_{1}$ and $R_{2}$ can be further optimised so that the optimised values would result in the large voltage variation as well as more linear characteristic curve within the smaller range of interest. Furthermore, we will consider using a new mirror with higher 
reflectance. This will obtain more linear voltage variation within shorter distance range than the current mirror.

In this paper, we compare performance of our contact force sensor with that of ATI Nano 17 in terms of dynamic force accuracy. However, this comparison is not enough to clarify various issues: linearity, repeatability, hysteresis, crosstalk, etc. Future work will consider a detailed study on such properties with improved calibration devices.

\section{B. Overall Size and Force Ranges of Contact Force Sensor}

Our prototype still has larger diameter and height $(D=26.5$ $\mathrm{mm}$ and $H=7.5 \mathrm{~mm}$ ) than that of the typical trocar sizes available in the market, which range from $10 \mathrm{~mm}$ to $15 \mathrm{~mm}$. We have designed our prototype with bigger diameter since we have to secure a space for wires. Every segment of the flexible manipulator requires at least five separate electrical wires: $V_{c c}$, ground, $V_{\text {out }}$ for three optoelectronic sensors. As the number of segment increases, the number of required wires increases proportionally, and this would make the manipulator impossible to be miniaturised. In order to resolve this issue, we could use a tiny ADC, ADS1015 (1.5 mm x 2.0 $\mathrm{mm} \times 0.4 \mathrm{~mm}$, X2QNFT, Texas Instrument, USA), for each segment and an $\mathrm{I}^{2} \mathrm{C}$ connector could be used to relay the connection from one segment to another, all the way to a microcontroller unit (MCU) which would be installed in the base of the manipulator.

Future work is to reduce the thickness of the metal ring and the mechanical sensor flexure supporting the outer ring and the inner ring. It is easy to find a high stiffness rigid metal to make the ring thinner, but it is hard to reduce the sensor mechanical flexure, the dyadic-S-shape beams. A new mechanical structure should be considered to miniaturise the overall size of the contact force sensor so that it fits within 10 to $15 \mathrm{~mm}$ diameter.

\section{Medical Safety and Sterilization}

The 3D printed stainless steel and plastic are biocompatible, hence, are safe for interaction with human organs or skins. Many medical devices have been fabricated by the same 3D printers and have been exploited across all medical fields [4445]. No electricity from the sensors and cables should flow through human organs and skins. Biocompatible silicone elastomer between the outer ring and the inner ring of the contact force sensor could be covered to guarantee safety and sterilization [46-47].

\section{Sensor Performance Limitations}

The proposed contact force sensor measures a net force applied to the contact force sensor. In general, the net force is a vector sum of all forces in presence. If the flexible arm is surrounded by multiple organs pressing the sensors from different angles at the same time, the proposed contact force sensor can measure the sum of all such forces. Detection of multiple contact points, measuring each force per point separately, requires alteration of the proposed structure and is left as a future work.
TABLE II. SENSOR ERROR PROPERTY

\begin{tabular}{|c|c|c|c|}
\hline Force & Range & $\begin{array}{c}\text { Average } \\
\text { Max/Min error }\end{array}$ & $\begin{array}{c}\text { RMS } \\
\text { error }\end{array}$ \\
\hline$F_{x}$ & $+/-4 \mathrm{~N}$ & $\begin{array}{r}+19.37 \% \pm 0.82, \\
-18.32 \% \pm 2.06\end{array}$ & $0.12 \mathrm{~N} \pm 0.0067$ \\
\hline$F_{y}$ & $+/-4 \mathrm{~N}$ & $\begin{array}{r}+18.56 \% \pm 1.69, \\
-17.00 \% \pm 1.32\end{array}$ & $0.11 \mathrm{~N} \pm 0.0032$ \\
\hline
\end{tabular}

\section{CONCLUSION AND FUTURE WORKS}

We presented development of the contact force sensor based on optoelectronic sensors and dyadic S-shape beams that can be integrated into flexible manipulators and verified the feasibility of the structure with experiments. We have observed the following remarks.

1. We proposed a new geometrical configuration of dyadic Sshape beams and simulated the configuration through Finite Element Analysis (FEA). We verified that the contact force components $F_{x}$ and $F_{y}$ can be accurately measured.

2. We optimised two resistors $R_{1}$ and $R_{2}$ for the optoelectronic sensors to produce large and linear voltage variation within the distance range of interest.

3. We fabricated the contact force sensor and verified that the force estimation is accurate as expected from the simulation.

4. We proposed the calibration method for the sensors using multiple linear regression.

The proposed mechanical structure was limited to guaranteeing to deform translationally, and the plastic used in the sensor structure had large hysteresis and it caused a deteriorated accuracy of the decoupling calibration matrix. Together with the wiring problem, we will consider other materials and a new sensor mechanical structure, and discuss corresponding admissible size of the flexible manipulator. Integrating a contact force sensor and shape sensor into a flexible manipulator is an important problem since then it can detect/adjust the overall curvature shape of the segmented body of the flexible manipulator according to environment of patient's internal body during surgery.

\section{REFERENCES}

[1] E. Degiannis, D. M. Bowley, and M. D. Smith, "Minimally invasive surgery in trauma: technology looking for an application," Injury, 35(5), pp. 474-478, 2004, doi: 10.1016/j.injury.2003.07.002.

[2] M. Siddaiah-Subramanya, K. W. Tiang, and M. Nyandowe, "A New Era of Minimally Invasive Surgery: Progress and Development of Major Technical Innovations in General Surgery Over the Last Decade," Surg Journal, 3(4), pp. e163-e166, 2017, doi: 10.1055/s0037-1608651.

[3] G. Watanabe, N. Ishikawa, "Da vinci surgical system. Kyobu geka. The Japanese journal of thoracic surgery," 67(8), pp. 686-689, 2014.

[4] R. Randell, N. Alvarado, S. Honey, J. Greenhalgh, P. Gardner, A. Gill, BA, D. Jayne, A. Kotze, A. Pearman, D. Dowding, "Impact of Robotic Surgery on Decision Making: Perspectives of Surgical Teams," AMIA Annu Symp Proc. pp. 1057-1066, 2015.

[5] R. A. Beasley, "Medical Robots: Current systems and research directions," J. Robot., vol. 2012, pp. 1-14, 2012. doi: $10.1155 / 2012 / 401613$. 
[6] S. Maeso, M. Reza, J. Mayol, J. B. M. Guerra, E. Andradas, and M. Plana, "Efficacy of the da vinci surgical system in abdominal surgery compared with that of laparoscopy: A systematic review and metaanalysis," Ann. Surgery, vol. 252, no. 2, pp. 254-262, 2010, doi: 10.1097/SLA.0b013e3181e6239e.

[7] G. Chirikjian and J. Burdick, "A hyper-redundant manipulator," IEEE Robot. Autom. Mag., vol. 1, no. 4, pp. 22-29, Dec. 1994, doi: $10.1109 / 100.388263$

[8] J. Burgner-Kahrs, D. C. Rucker, H. Choset, "Continuum Robots for Medical Applications: A Survey," IEEE Trans. on Robotics, vol. 31, no. 6, pp. 1261-1280, 2015, doi: 10.1109/TRO.2015.2489500.

[9] R. Grassmann, V. Modes, J. Burgner-Kahrs, "Learning the Forward and Inverse Kinematics of a 6-DOF Concentric-Tube Continuum Robot in SE(3)," IEEE/RSJ International Conference on Intelligent Robots and Systems, pp. 5125-5132, 2018, doi:10.1109/IROS.2018.8594451.

[10] J. Starke, E. Amanov, M.T. Chikhaoui, J. Burgner-Kahrs, "On the Merits of Helical Tendon Routing in Continuum Robots," IEEE/RSJ International Conference on Intelligent Robots and Systems, pp. 64706476, 2017, doi: 10.1109/IROS.2017.8206554.

[11] T. Ota, A. Degani, D. Schwartzman, B. Zubiate, J. McGarvey, H. Choset, M. A. Zenati, "A Highly Articulated Robotic Surgical System for Minimally Invasive Surgery,” Ann Thorac Surg. 2009 April ; 87(4): pp.1253-1256. doi:10.1016/j.athoracsur.2008.10.026

[12] Hansen Medical Inc, http://hansenmedical.com/us/en, 14 ${ }^{\text {th }}$ Oct, 2018.

[13] H. B. Gilbert, D. C. Rucker, and R. J. Webster III, "Concentric tube robots: The state of the art and future directions," in Proc. Int. Symp. Robot. Res.,pp. 1-16, 2013, doi: 10.1007/978-3-319-28872-7 15 .

[14] K.W. Kwok, K. Hung Tsoi, V. Vitiello, J. Clark, G. C. T. Chow, W. Luk, and G.-Z. Yang, "Dimensionality reduction in controlling articulated snake robot for endoscopy under dynamic active constraints," IEEE Trans. Robot., vol. 29, no. 1, pp. 15-31, Feb. 2013, doi: 10.1109/TRO.2012.2226382.

[15] J. Fraś, J. Czarnowski, M. Maciaś, J. Główka, M. Cianchetti, A Menciassi, "New STIFF-FLOP module construction idea for improved actuation and sensing, "2015 IEEE International Conference on Robotics and Automation (ICRA), pp.2901-2906, 2015, doi: 10.1109/ICRA.2015.7139595.

[16] A. Arezzo, Y. Mintz, M. E. Allaix, G. Gerboni, M. Brancadoro, M. Cianchetti, A. Menciassi, H. Wurdemann, Y. Noh, J. Fras, J. Glowka Z. Nawrat, G. Cassidy, R. Walker, S. Arolfo, M. Bonino, M. Morino, K. Althoefer, "Total Mesorectal Excision using a soft and flexible robotic arm: a feasibility study in cadaver models, Surgical Endoscopy and Other Interventional Techniques,” 31(1), pp. 264-273, 2017, doi: 10.1007/s00464-016-4967-x.

[17] Y. Noh, S. Sareh, H. A. Würdemann, H. Liu, J. Housden, K. Rhode, K. Althoefer, "Three-axis Fiber-optic Body Force Sensor for Flexible Manipulators," IEEE Sensors Journal, 16(6),7297802, pp. 1641-1651, 2016, doi: 10.1109/JSEN.2015.2488099.

[18] E. Ayvali, C.-P. Liang, M. Ho, Y. Chen, and J. P. Desai, "Towards a discretely actuated steerable cannula for diagnostic and therapeutic procedures,” Int. J. Robot. Res., vol. 31, no. 5, pp. 588-603, 2012, doi: $10.1177 / 0278364912442429$.

[19] Y. Noh, J. Bimbo, S. Sareh, H. Wurdemann, J. Fraś, H. Liu, J. Housden, K. Althoefer, K. Rhode, "Multi-Axis force/torque sensor based on Simply-Supported beam and optoelectronics," MDPI Sensors, 16(11), 1936, 2016, doi: 10.3390/s16111936.

[20] Y. Noh, E.L. Secco, S. Sareh, H.A. Wurdemann, H. Liu, K. Althoefer, "A continuum body force sensor designed for flexible surgical robotic devices," IEEE Engineering in Medicine and Biology Society (EMBC 2014), pp.3711-3714, 2014, doi: 10.1109/EMBC.2014.6944429.

[21] X. Ma, P. W. Chiu, Z. Li, "Shape Sensing of Flexible Manipulators with Visual Occlusion based on Bezier Curve," IEEE Sensors Journal, pp.8133-8142, 2018, doi: 10.1109/JSEN.2018.2862925.

[22] X. Ma, P. W. CHIU, and Z. LI, "Real-time Deformation Sensing for Flexible Manipulators with Bending and Twisting," IEEE Sensors Journal, pp.6412-6422, 2018, doi: 10.1109/JSEN.2018.2846762.

[23] Y. Noh, S. Sareh, J. Back, H.A. Wurdemann, T. Ranzani, E.L. Secco, A. Faragasso, H. Liu, K. Althoefer, "Three-axial body force sensor for flexible manipulators," IEEE International Conference on Robotics and Automation (ICRA 2014), pp.6388-6393, 2014, doi: 10.1109/ICRA.2014.6907802.

[24] S. Sareh, A. Jiang, A. Faragasso, Y. Noh, T. Nanayakkara, P. Dasgupta, L.D. Seneviratne, H.A. Wurdemann, K. Althoefer, "MR-Compatible bio-inspired tactile sensor sleeve for surgical soft manipulators," IEEE
International Conference on Robotics and Automation (ICRA 2014) pp.1454 - 1459 2014, doi: 10.1109/ICRA.2014.6907043.

[25] S. Sefati, R. J. Murphy, F. Alambeigi, M. Pozin, I. Iordachita, R. H. Taylor, M. Armand, "FBG-Based Control of a Continuum Manipulator Interacting With Obstacles," 2018 IEEE/RSJ International Conference on Intelligent Robots and Systems, 2018, doi: 10.1109/IROS.2018.8594407

[26] Y. He, L. Zhu, G. Sun, M. Yu, M. Dong, "Design, Measurement and Shape Reconstruction of Soft Surgical Actuator Based on Fiber Bragg Gratings,"Appl. Sci. 2018, vol.8, no.10, 1773; doi:10.3390/app8101773.

[27] S. C. Ryu, P. E. Dupont, "FBG-based shape sensing tubes for continuum robots," IEEE International Conference on Robotics and Automation, pp. 3531-3537, 2014, doi:10.1109/ICRA.2014.6907368.

[28] S. Thielmann, U. Seibold, R. Haslinger, G. Passig, T. Bahls, S. Jorg, M. Nickl, A. Nothhelfer, U. Hagn, G. Hirzinger, "MICA - A new generation of versatile instruments in robotic surgery," IEEE/RSJ International Conference on Intelligent Robots and Systems (IROS), pp. 871-878. 2010, doi: 10.1109/IROS.2010.5649984.

[29] S. Sareh, Y. Noh, M. Li, T. Ranzani, H. Liu, K. Althoefer, "Macrobend optical sensing for pose measurement in soft robot arms," Smart Materials and Structures, 24(12),125024, 2015, doi: 10.1088/0964$1726 / 24 / 12 / 125024$.

[30] A. M. Aizzuddin, Z. M. Hafizi, L. V. Kee, E. Vorathin, K. S. Lim, "Development of Fibre Bragg grating (FBG) dynamic pressure transducer with diminutive voltage inconsistency," IOP Conf. Series: Materials Science and Engineering 257 (2017) 012080 doi:10.1088/1757-899X/257/1/012080.

[31] T. Jeong, S. Han, K. Rhode, Y. Noh, "New shape sensing approach for flexible manipulator used in MIS," BioMedEng 2018.

[32] J. H. B. Koh, T. Jeong, S. Han, W. Li, K. Rhode, Y. Noh, "Optoelectronic Sensor-based Shape Sensing Approach for Flexible Manipulators," New shape sensing approach for flexible manipulator used in MIS," 14th International Engineering in Medicine and Biology Conference (EMBC 2019), 2019.

[33] P. Gawenda, Y. Noh, J. Fras, S. Han, S. Wang, R. Housden and K. Rhode, "Contact force sensor for flexible manipulators for MIS (minimally invasive surgery)," 7th Joint Workshop on New Technologies for Computer/Robot Assisted Surgery, 2017.

[34] C. E. Reiley, T. Akinbiyi, D. Burschka, D. C. Chang, A. M. Okamura, and D. D. Yuh, "Effects of visual force feedback on robot-assisted surgical task performance," J. Thoracic Cardiovascular Surgery, vol. 135, no. 1, pp. 196-202, Jan. 2008, doi: 10.1016/j.jtcvs.2007.08.043.

[35] C. R. Wagner, N. Stylopoulos, P. G. Jackson, and R. D. Howe, "The benefit of force feedback in surgery: Examination of blunt dissection," Presence, Teleoper. Virtual Environ., vol. 16, no. 3, pp. 252-262, 2007, doi: 10.1162 pres.16.3.252.

[36] M. Kitagawa, D. Dokko, A. M. Okamura, and D. D. Yuh, "Effect of sensory substitution on suture-manipulation forces for robotic surgical systems," J. Thoracic Cardiovascular Surgery, vol. 129, no. 1, pp. 151158, Jan. 2005, doi: 10.1016/j.jtcvs.2004.05.029.

[37] S. Petrache, D. Duminică, C. L. Adrian, L. Bogatu, "A New Method for Establishing the Performances of an Optoelectronic Sensor," Conference: International Conference of Mechatronics and CyberMixmechatronics, pp.204-219, 2017, doi: 10.1007/978-3-319-63091524.

[38] G. Palli, M. Hosseini, C. Melchiorri, "A Simple and Easy-to-Build Optoelectronics Force Sensor Based on Light Fork: Design Comparison and Experimental Evaluation," Sensors and Actuators A Physical 269, 2017, doi: 10.1016/j.sna.2017.04.054.

[39] C. Melchiorri, L. Moriello, G. Palli and U. Scarcia, "A New Force/Torque Sensor for Robotic Applications Based on Optoelectronic Components," 2014 IEEE International Conference on Robotics \& Automation (ICRA), pp.6408-6413, 2014, doi: 10.1109/ICRA.2014.6907805.

[40] L. L. Nathans, F. L. Oswald, and K. Nimon, "Interpreting multiple linear regression: A guidebook of variable importance," Pract. Assessment, Res. Eval., vol. 17, no. 9, pp. 1-19, 2012, doi: 10.4159/harvard.9780674063297.c1.

[41] Jiantao Yao, Hongyu Zhang, Ximei Xiang, Huidong Bai, Yongsheng Zhao, "A 3-D printed redundant six-component force sensor with eight parallel limbs," Sensors and Actuators A: Physical, A 247, pp. 90-97, 2016, doi: 10.1016/j.sna.2016.05.041 
[42] M. Kang, S. Lee, J. Kim, "Shape optimization of a mechanically decoupled six-axis force/torque sensor," Sensors and Actuators A209 (2014) 41-51, doi: 10.1016/j.sna.2014.01.001.

[43] Y. Noh, L. Lindenroth, S. Wang, R. J. Housden, A. v. Wingerden, W Li, K. Rhode, A 2-piece six-axis force/torque sensor capable of measuring loads applied to tools of complex shapes, 2019 IEEE/RSJ International Conference on Intelligent Robots and Systems (IROS 2019), 2019.

[44] Materialise NV, Belgium, https://www.materialise.com. 20 $0^{\text {th }}$ Aug, 2019.

[45] 3D Systems Corporation, USA, "3D Systems Expands Class VI Certified Materials To ProJet ${ }^{\mathrm{TM}}$ Series - Expands Its Healthcare Solutions Reach With Biocompatible Materials," Retrieved from https://uk.3dsystems.com, $20^{\text {th }}$ Aug, 2019.

[46] L. E. Diment, M. S. Thompson, J. H. M. Bergmann, "Clinical efficacy and effectiveness of 3D printing: a systematic review," BMJ Open., vol. 7, no. 12, e016891, doi: 10.1136/bmjopen-2017-016891.

[47] P. V. Mohanan, K. Rathinam, "Biocompatibility studies on silicone rubber," 14th International Engineering in Medicine and Biology Conference (EMBC 1995), 1995, doi: 10.1109/RCEMBS.1995.533005.

[48] V. Vince, M. A. Thil, C. Veraart, I. M. Collin, J. Delbeke, "Biocompatibility of platinum-metallized silicone rubber: in vivo and in vitro evaluation," Journal of Biomaterials Science Polymer Edition, vol.15, no.2, pp.173-88, 2004, doi: 10.1163/156856204322793566.

[49] Sareh, S. and Noh, Y (2018) Low profile stretch sensor for soft wearable robotics, IEEE International Conference on Soft Robotics (RoboSoft 2018), 2018, doi: 10.1109/ROBOSOFT.2018.8405372.

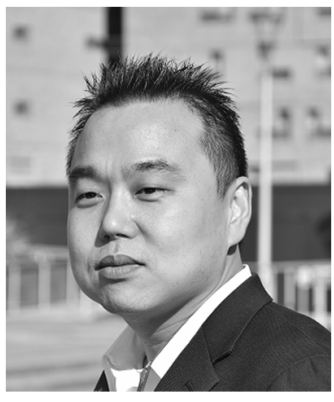

Yohan Noh received the B.S. degree from the Department of Mechanical Engineering, Seoul National University of Science and Technology, South Korea, in 2002, the B.S. degree from the Department of Electrical Engineering, Yonsei University, South Korea, in 2004, and the M.S. and Ph.D. degrees from the Department of Science and Engineering, Waseda University, Tokyo, Japan, in 2007 and 2011, respectively. He worked as a Research Associate for Robotics Research in the Department of Biomedical Engineering, King's College London 2013 to 2019. Currently, he is working as a lecturer in the Department of Mechanical and Aerospace Engineering, Brunel University London. His research interests include development of force and tactile sensors, haptics, robot assisted ultrasound diagnostic system, medical training system, and medical robots.

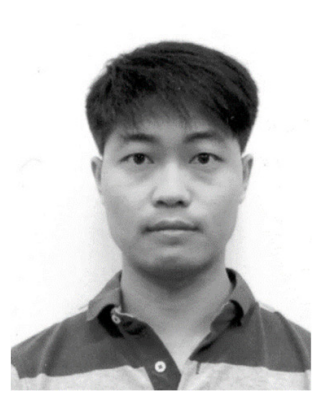

Sangin Han received the M.S. degree from University of Maryland, College Park, MD, and $\mathrm{Ph} . \mathrm{D}$ degree from Texas $\mathrm{A} \& \mathrm{M}$ University, College Station, TX, U.S.A. in 2014 and 2019, respectively. His research interests include linear systems control, PID control, and robotics applications such as UAVs and ventral camera gimbals. He is currently working in U. S. Army Research Laboratory, Adelphi, MD, and Booz Allen Hamilton Inc., McLean, VA, U.S.A.

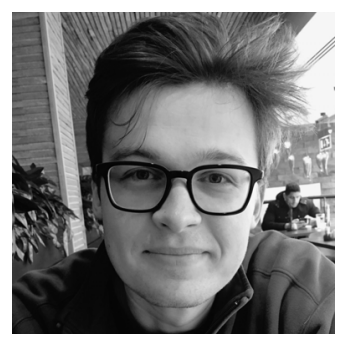

Pawel Gawenda Received MEng degree in Biomedical Engineering at King's College London in 2018. Currently working as a Data Analyst in FinTech industry.

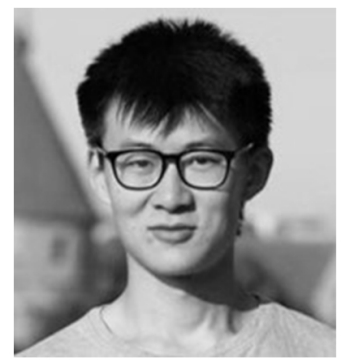

Wanlin Li received the B.Eng. degree in electronic engineering from Queen Mary University of London, UK, in 2016, where he is currently pursuing the Ph.D. degree in robotics. His current research interests include development of force and tactile sensors.

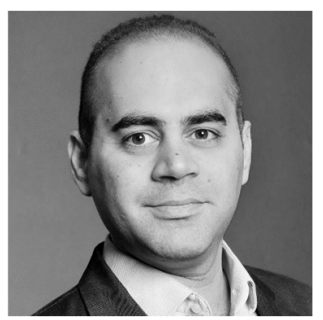

Sina Sareh received the B.S. degree in electrical engineering from Tehran Polytechnic, Iran, in 2005, the M.S. degree in control systems from the University of Sheffield, U.K., in 2007, and the Ph.D. degree in robotics from the University of Bristol, U.K., in 2012. Dr Sareh is the Academic Leader in Robotics at Royal College of Art. $\mathrm{He}$ is currently a Reader in Robotics and Design Intelligence and a Fellow of the Engineering and Physical Sciences Research Council (EPSRC) whose research develops new robotic solutions to human safety, access and performance issues in medical and industrial operations.

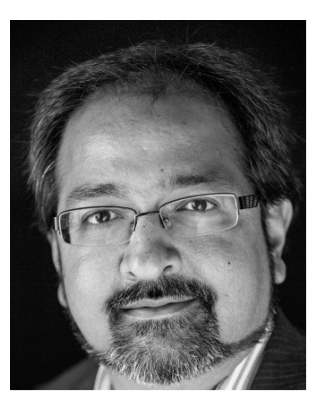

Kawal Rhode received his bachelor's degree in Basic Medical Sciences and Radiological Sciences from King's College London in 1992 and his Ph.D. degree from the Department of Surgery, University College London in 2001. From 2001 to 2007, he carried research within the School of Biomedical Engineering \& Imaging Sciences, King's College London where he is currently Professor of Biomedical Engineering and Head of Education. His research interests include image-guided interventions, biophysical modeling, computer simulation of minimally invasive procedures, medical robotics, AR \& VR for healthcare applications and 3D printing for medical applications. Professor Rhode has more than 350 publications in the field of biomedical engineering. 